\title{
ARTICLE
}

Received 5 Nov 2016 | Accepted 20 Mar 2017 | Published 31 May 2017

DOI: $10.1038 /$ ncomms15314

OPEN

\section{Engineered clearing agents for the selective depletion of antigen-specific antibodies}

Siva Charan Devanaboyina ${ }^{1,2}$, Priyanka Khare ${ }^{1,2}$, Dilip K. Challa ${ }^{1,2}$, Raimund J. Ober ${ }^{1,3} \&$ E. Sally Ward ${ }^{1,2}$

Here we have designed a novel class of engineered antibody-based reagents ('Seldegs') that induce the selective degradation of antigen-specific antibodies. We demonstrate the rapid and specific clearance of antibodies recognizing the autoantigen, myelin oligodendrocyte glycoprotein and tumour target, HER2. Seldegs have considerable potential in multiple areas, including the treatment of antibody-mediated autoimmunity and diagnostic imaging.

\footnotetext{
${ }^{1}$ Department of Molecular and Cellular Medicine, Texas A\&M University Health Science Center, 469 Joe H. Reynolds Medical Sciences Building, 1114 TAMU, College Station, Texas 77843, USA. ${ }^{2}$ Department of Microbial Pathogenesis and Immunology, Texas A\&M University Health Science Center, 3107 Medical Research \& Education Building, 8447 State Highway 47, Bryan, Texas 77807, USA. ${ }^{3}$ Department of Biomedical Engineering, Texas A\&M University, 5045 Emerging Technologies Building, 3120 TAMU, College Station, Texas 77843, USA. Correspondence and requests for materials should be addressed to R.J.O. (email: raimund.ober@tamu.edu) or to E.S.W. (email: sally.ward@tamu.edu).
} 
T he development of strategies to specifically decrease antigen-specific antibody levels for the clearance of deleterious antibodies during therapy and diagnosis represents a longstanding and unresolved challenge. Such an approach would have broad utility in areas such as the treatment of antibody-mediated autoimmunity, antibody-mediated transplant rejection and the clearance of background during diagnostic imaging. The knowledge that the neonatal Fc receptor, FcRn, maintains immunoglobulin $\mathrm{G}(\mathrm{IgG})$ levels and transport in the body has prompted the development of antibody- or peptidebased inhibitors to reduce $\mathrm{IgG}$ levels ${ }^{1-5}$. However, these inhibitors block the interaction of the Fc region of $\operatorname{IgG}$ with FcRn and decrease levels of IgGs of all specificities, including protective antibodies. To overcome these off-target effects, here we have designed a novel class of engineered antibodies that selectively clear antigen-specific antibodies without modulating the levels of antibodies of other specificities. We have named these agents 'Seldegs' to indicate their ability to selectively degrade antibodies of defined specificities.

The design of clearing agents for antigen-specific antibodies presents several challenges: first, antigen-specific antibody levels are typically very low compared with those of antibodies of irrelevant specificities, and these two antibody pools are similar with shared constant regions but distinct variable domains. Second, since the antibodies being targeted are bivalent, crosslinking could result in inflammatory immune complexes. Seldegs were therefore designed to display recombinant antigen as a monomer linked to a dimeric, human IgG1-derived Fc fragment using a similar approach to that described previously for monomeric erythropoietin (Epo)-Fc fusions ${ }^{6}$. Mutations to ablate interactions with human $\mathrm{Fc} \gamma \mathrm{Rs}^{7}$ and enhance binding to FcRn in the $\mathrm{pH}$ range 6.0-7.4 (ref. 1) were inserted. Naturally occurring IgGs have substantially higher affinity for FcRn at acidic $\mathrm{pH}$ than at near neutral $\mathrm{pH}$, and this property is essential for the recycling and transcytosis of IgG within FcRn-expressing cells ${ }^{8}$. By contrast, gain of binding affinity of an $\mathrm{Fc}$ (or $\mathrm{IgG}$ ) for $\mathrm{FcRn}$ at $\mathrm{pH} 7.4$ results in receptor-mediated internalization into cells and lysosomal delivery, $, 9,10$. In the current study, we demonstrate that $\mathrm{Fc}$-antigen fusions, or Seldegs, containing such affinity-enhanced Fc fragments selectively capture antigenspecific antibodies and direct them into degradative lysosomal compartments in FcRn-expressing cells.

\section{Results}

Clearance of antigen-specific antibodies by Seldegs. To demonstrate the generality of the Seldeg approach, antibodies specific for two antigens were targeted (Fig. 1a): first, the extracellular domain (ECD) of myelin oligodendrocyte glycoprotein (MOG-Seldeg), which is recognized by autoreactive antibodies in both animal models of multiple sclerosis and multiple sclerosis in humans ${ }^{11-13}$. Second, the ECD of HER2 (HER2-Seldeg), a welldefined target for therapy and diagnostic imaging of HER2overexpressing tumours with HER2-specific antibodies such as trastuzumab (TZB) $)^{14}$. Both MOG- and HER2-Seldegs have the expected binding properties for FcRn, MOG-specific antibody (8-18C5 (ref. 15)) and TZB (Fig. 1b; Supplementary Fig. 1; Supplementary Table 1). The expression yields of the Seldegs are $\sim 50 \mathrm{mgl}^{-1}$ (MOG-Seldeg) and $\sim 15 \mathrm{mgl}^{-1}$ (HER2-Seldeg). SDS-polyacrylamide gel electrophoresis and high-performance liquid chromatography analyses also indicate that these Fc fusion proteins have favourable biophysical properties following storage at $4{ }^{\circ} \mathrm{C}$ (30 days) or $37^{\circ} \mathrm{C}$ (5 days) in PBS or human serum (Supplementary Fig. 2). In addition, the Seldegs retain their affinity for binding to $8-18 \mathrm{C} 5\left(K_{\mathrm{D}}=33.0 \mathrm{nM}\right.$; MOG-Seldeg $)$ and TZB $\left(K_{\mathrm{D}}=14.3 \mathrm{nM}\right.$; HER2-Seldeg $)$ following incubation for 5 days at $37^{\circ} \mathrm{C}$.
We first investigated the ability of Seldegs to clear antigenspecific antibodies in transgenic mice that express human Fc $\gamma$ Rs $\left(\right.$ huFc $\gamma \mathrm{R}$ mice) ${ }^{16}$. These mice were used since Seldegs have human IgG1-derived Fc regions. Mice were injected with ${ }^{125}$ I-labelled, MOG-specific antibody 8-18C5 (ref. 15). Twentyfour hours later, MOG-Seldeg was injected at a 16-fold (high dose) or 4-fold (low dose) molar excess over target. The delivery of MOG-Seldeg resulted in a rapid decrease in 8-18C5 levels in the blood and whole body (Fig. 1c). Importantly, the total IgG levels in serum of mice before and $48 \mathrm{~h}$ following treatment with high or low dose of Seldeg were not significantly different (Supplementary Fig. 3), indicating the selectivity of Seldegmediated clearance. In addition, injection of an analogous construct without the FcRn-enhancing MST-HN mutations (MOG-WT) had no effect on 8-18C5 clearance (Fig. 1c).

To further analyse the specificity of Seldegs and their effect on antibodies with different antigen recognition properties, the behaviour of the HER2-specific humanized antibody TZB ${ }^{14}$ was investigated in the presence of HER2-Seldeg and MOG-Seldeg (Fig. 1d). HuFc $\gamma$ R mice were injected with ${ }^{125}$ I-labelled TZB and subsequently with a 4 -fold molar excess of Seldeg. As controls, equivalent molar amounts of HER2-WT (analogous to HER2Seldeg without FcRn-enhancing mutations), MOG-Seldeg or hen egg lysozyme-specific human IgG1 with the MST-HN mutations $(\mathrm{Abdeg})^{1}$ were used. This Abdeg contains the same mutations to increase FcRn binding as those in the Seldegs. HER2-Seldeg induced a decrease in TZB levels in the blood and whole body, whereas the control proteins resulted in similar behaviour to that observed for vehicle (PBS; Fig. 1d). Remarkably, blood levels of TZB were reduced to $\sim 30 \%$ of the injected dose within $2 \mathrm{~h}$ following Seldeg delivery (Fig. 1e).

Treatment with MOG- and HER2-Seldeg resulted in biphasic clearance of the targeted radiolabelled antibodies (Fig. 1c,d). Within the first $\sim 50 \mathrm{~h}$ of Seldeg delivery, the radiolabelled antibodies are rapidly cleared. This is followed by slower clearance rates that are close to those in control animals (Fig. 1d). Analyses of the pharmacokinetic behaviour of MOG- and HER2-Seldeg revealed relatively short $\beta$-phase halflives (MOG-Seldeg, $47.0 \pm 1.4 \mathrm{~h}$ (s.d.); HER2-Seldeg, $38.3 \pm 3.1 \mathrm{~h}$; $n=5$ for both groups) that were similar to those of the Abdeg $(37.8 \pm 2.5 \mathrm{~h} ; n=5$; Supplementary Fig. 4). For comparative purposes, we also determined the $\beta$-phase half-lives of MOG- and HER2-WT $(102.3 \pm 22.0$ and $87.2 \pm 11.9$ h, respectively; $n=5$ for both groups). The rapid clearance of Seldegs is consistent with earlier studies demonstrating that engineered antibodies with increased binding to $\mathrm{FcRn}$ at near neutral $\mathrm{pH}$ results in shorter in vivo half-lives $8,17,18$. This behaviour indicates that the persistence of a low percentage of $8-18 \mathrm{C} 5$ or TZB following $\sim 50 \mathrm{~h}$ of treatment is due to reduced levels of Seldeg, resulting in incomplete capture of the targeted antibodies.

Seldegs internalize targeted antibodies into lysosomes. To investigate the mechanism of Seldeg activity at the cellular level, we analysed their effects on the internalization and accumulation of 8-18C5 and TZB in endothelial cells (HMEC-1, human microvasculature endothelial cells) transfected with a human FcRn-GFP expression construct (mutated to confer binding properties analogous to those of mouse $\mathrm{FcRn}^{19}$ ). Seldegs efficiently internalize cognate antibodies into endosomes within cells (Fig. 2a-c). Importantly, high levels of $8-18 \mathrm{C} 5$ or TZB in cells result from treatment with MOG- or HER2-Seldeg, respectively, and the majority of targeted antibody is retained by the cells during a 60 min chase (Fig. 2a). By contrast, target antibody accumulation within cells is substantially lower in the presence of MOG-WT (8-18C5) or HER2-WT (TZB), and the 
a

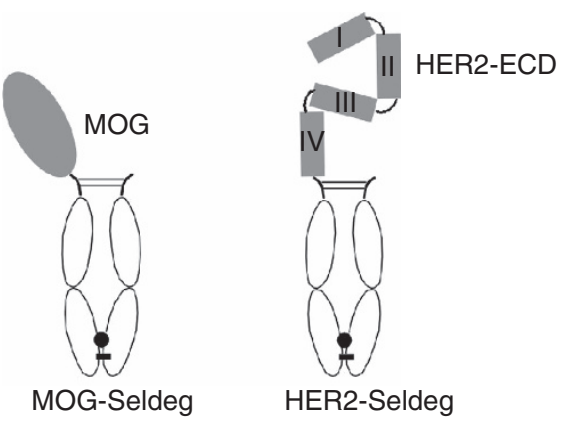

C

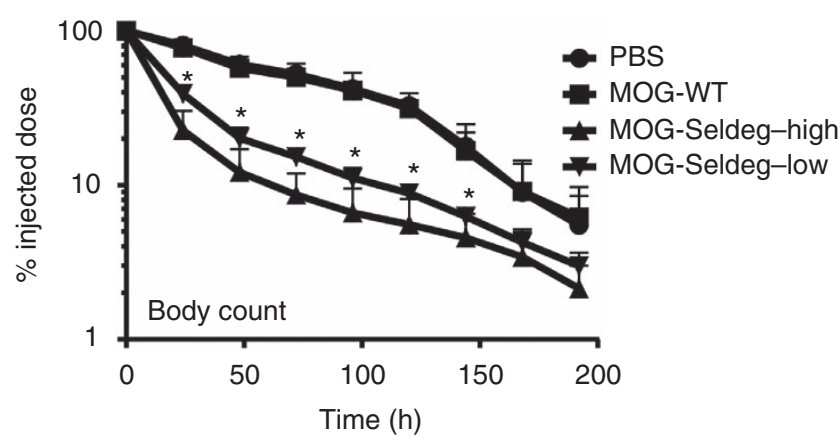

d
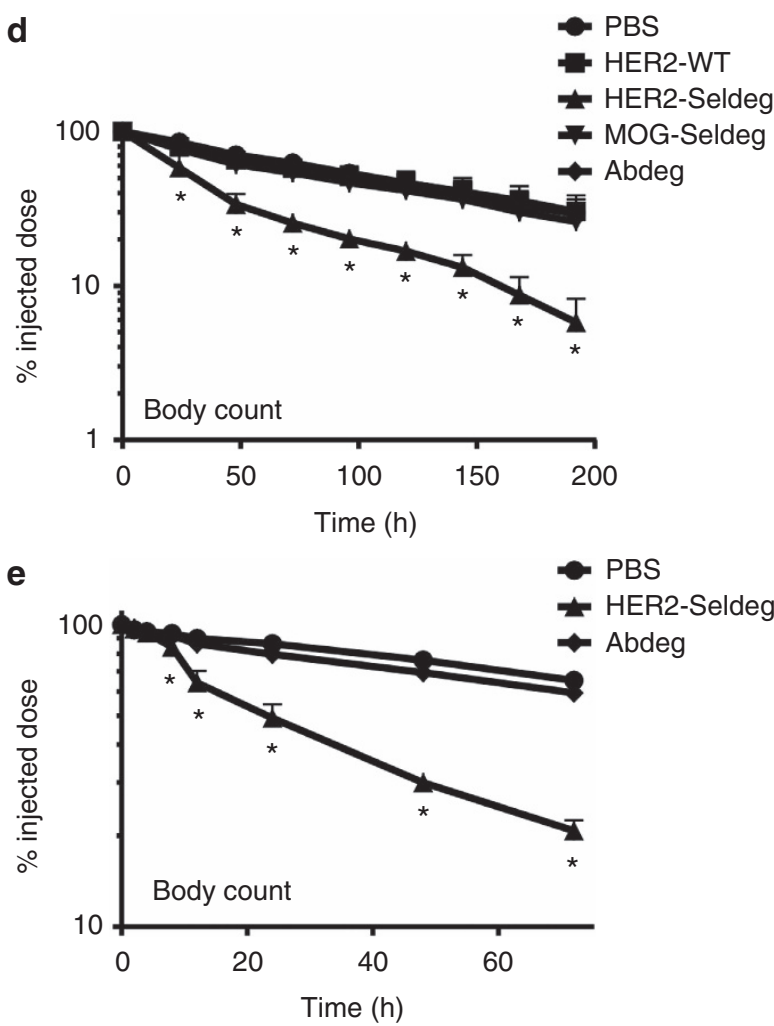

internalized antibody is efficiently recycled over $60 \mathrm{~min}$ (Fig. 2a). Following $8 \mathrm{~h}$ incubation, the targeted antibody and corresponding Seldeg are delivered to lysosomes (Fig. 2d; b
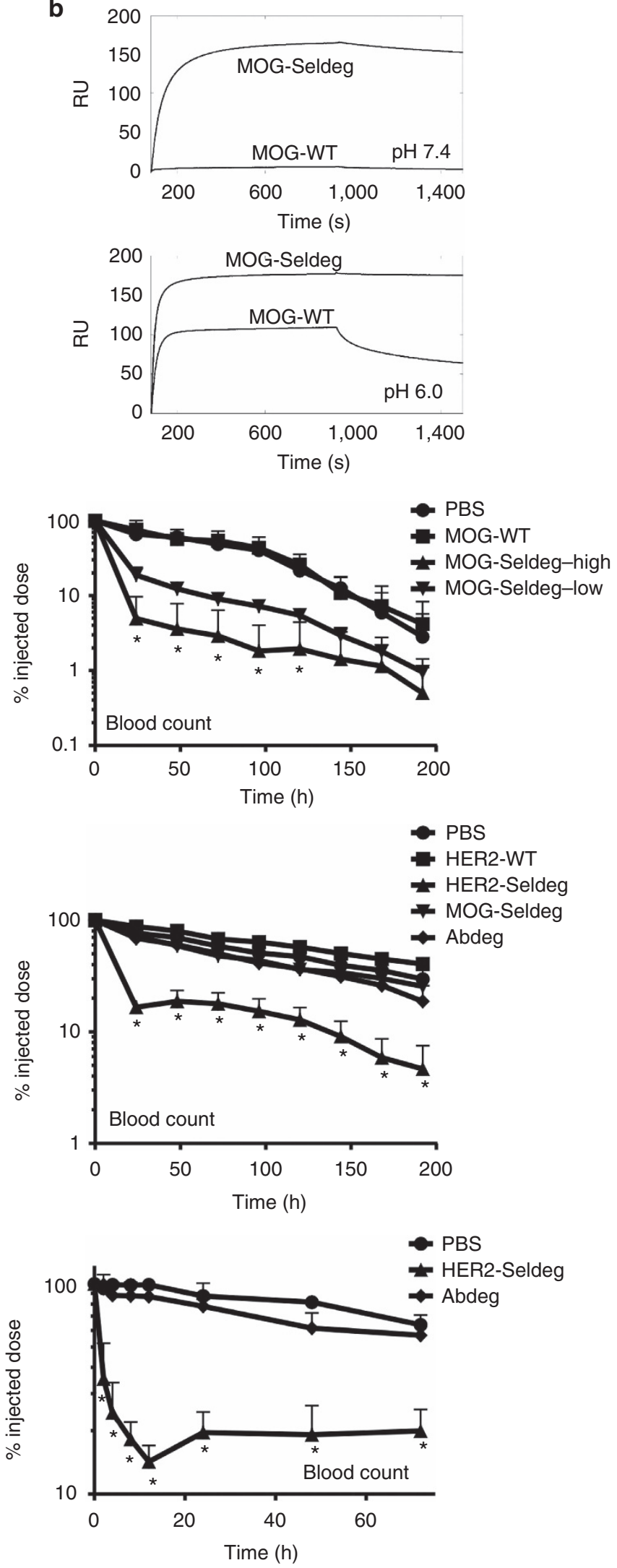

Supplementary Fig. 5). Importantly, cells do not accumulate 8-18C5 and TZB in lysosomes in the presence of HER2-Seldeg or MOG-Seldeg, respectively (Fig. 2d; Supplementary Fig. 5). 
a

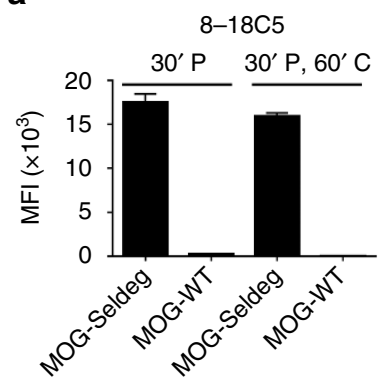

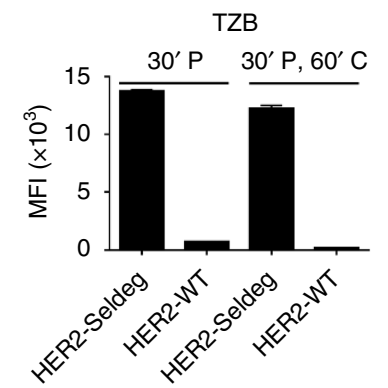

b

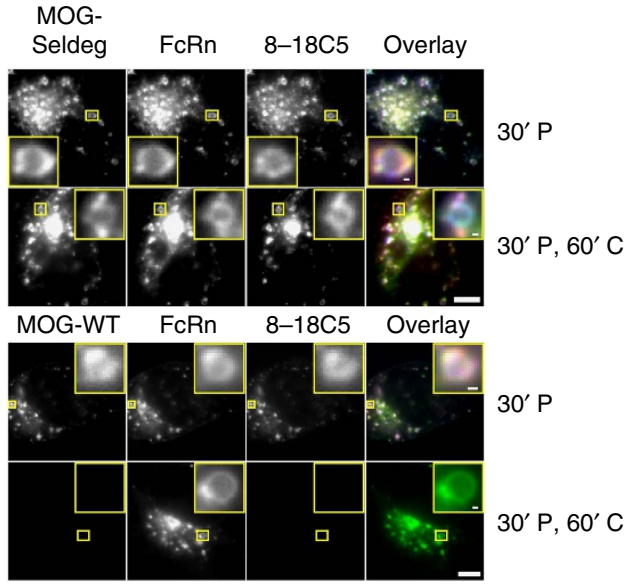

C

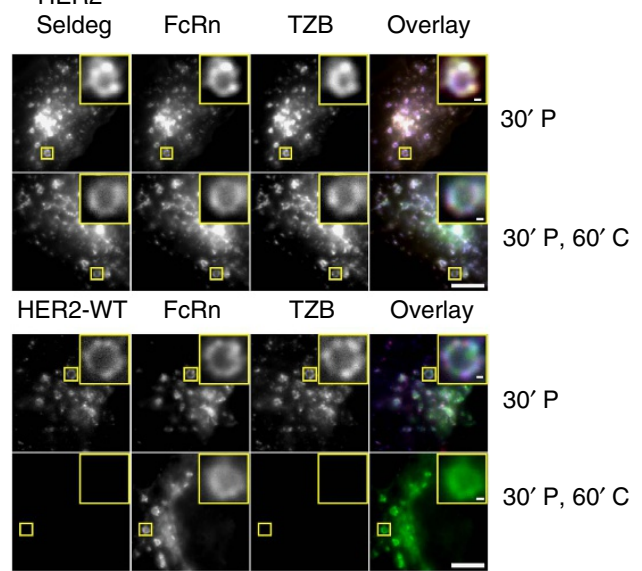

d

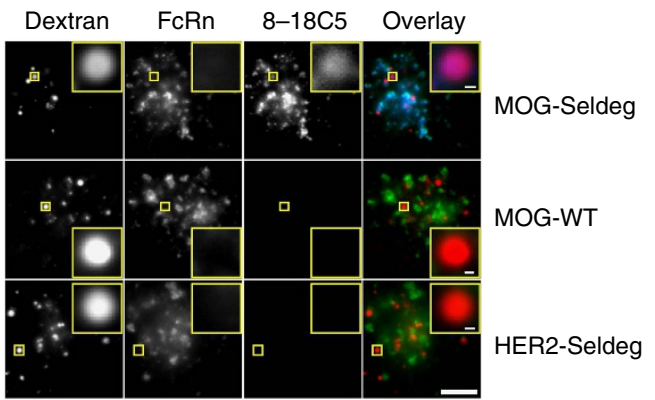

Figure 2 | Seldegs increase the accumulation of antigen-specific antibodies in human endothelial (HMEC-1) cells expressing FcRn-GFP. (a) HMEC-1 cells were incubated with $100 \mathrm{nM}$ Alexa 647-labelled 8-18C5 (MOG-specific) or TZB (HER2-specific) in complex with 400 nM MOG-Seldeg/MOG-WT or HER2-Seldeg/HER2-WT for 30 min and chased for 0 (30' P) or 60 min (30' P, 60' C). Mean fluorescence intensities (MFI) of Alexa 647-labelled 8-18C5 or TZB for triplicate samples were determined by flow cytometry. Error bars indicate s.d. (b,c) HMEC-1 cells were plated on coverslips and treated as in $\mathbf{a}$, except that Seldegs or control WT proteins were labelled with Alexa 555 and cells were fixed for microscopy. Images of representative cells from multiple cells analysed are shown with GFP, Alexa 555 and Alexa 647 in overlays pseudocoloured green, red and blue, respectively. Representative endosomes in the insets are cropped and expanded. (d) HMEC-1 cells were pre-pulsed with Alexa 555-labelled dextran for $2 \mathrm{~h}$, washed and pulsed with 8-18C5 in complex with MOG-Seldeg, MOG-WT and HER2-Seldeg (concentrations and labels as for a) for $30 \mathrm{~min}$, followed by an $8 \mathrm{~h}$ chase. Cells were washed, fixed and imaged, and images for a representative cell from multiple cells analysed are presented. Representative lysosomes in the insets are cropped and expanded. For the overlay, GFP, Alexa 555 and Alexa 647 are pseudocoloured as in $\mathbf{b}$. For $\mathbf{b}-\mathbf{d}$, scale bars $=5 \mu \mathrm{m}$, and for insets, scale bars $=0.25 \mu \mathrm{m}$. Data shown are representative of at least two independent experiments.

In summary, we have demonstrated that engineered antigen-Fc fusion proteins (Seldegs) induce rapid and substantial decreases of antigen-specific antibody levels. Further, Seldegs are effective at relatively low doses that, by contrast with earlier approaches ${ }^{1-5}$, do not alter the clearance of antibodies of non-targeted specificities. The properties of Seldegs indicate their potential applications in multiple clinical situations where it is desirable to selectively clear antibodies of defined specificities.

\section{Methods}

Cell lines. HMEC-1 cells, a generous gift from Francisco Candal (CDC), were maintained in phenol red-free HAMS F12-K medium (US Biological). BT-474 (American Type Culture Collection; HTB-20) were maintained according to the supplier's protocol. Cell lines were tested at monthly intervals for mycoplasma contamination and were authenticated by short tandem repeat analysis.

Generation of expression constructs. The gene encoding the HER2 leader peptide and ECD (consisting of 629 residues) was isolated from a HER2-overexpressing breast cancer cell line (BT-474) employing standard molecular biology techniques. This gene was fused via a IEGRMD linker peptide to the $\mathrm{N}$ terminus of the hinge region of a gene encoding the human IgG1-derived $\mathrm{Fc}$ fragment using splicing by overlap extension ${ }^{20}$. Mutations to ablate binding to Fc $\gamma$ Rs (G236R/ L328R; EU numbering) ${ }^{7}$, enhance binding to FcRn (MST-HN; M252Y/S254T/ $\mathrm{T} 256 \mathrm{E} / \mathrm{H} 433 \mathrm{~K} / \mathrm{N} 434 \mathrm{~F})^{1}$ and generate 'knobs-into-holes' (Y349T/T394F) 21,22 were inserted into the $\mathrm{Fc}$ fragment gene using standard methods. Cysteine (C220) in the hinge region that bridges with cysteine in the light-chain constant region was also mutated to serine. $\mathrm{Fc}$ fragment genes without fused antigen were generated with complementary knobs-into-holes mutations $(\mathrm{S} 364 \mathrm{H} / \mathrm{F} 405 \mathrm{~A})^{21,22}$. Similar methodology as described above was used to produce a fusion construct encoding

Figure 1 | TZB and 8-18C5 are rapidly cleared by Seldegs in transgenic mice expressing huFc $\gamma$ Rs. (a) Schematic representation of Seldeg design. (b) A concentration of $100 \mathrm{nM} \mathrm{MOG-Seldeg}$ or MOG-WT was injected over immobilized mouse FcRn at the indicated pH. (c) Mice were intravenously injected with radiolabelled $\left({ }^{125} \mathrm{l}\right)$ 8-18C5 (15 $\left.\mu \mathrm{g}\right)$ and $24 \mathrm{~h}$ later PBS, MOG-WT (31 $\left.\mu \mathrm{g}\right)$ or MOG-Seldeg (4-fold (31 $\left.\mu \mathrm{g}\right)$ or 16-fold (125 $\mu \mathrm{g}$ ) molar excess; low or high dose, respectively) was delivered intravenously. Radioactivity levels were determined at the indicated times. Whole body or blood levels obtained immediately before Seldeg or control delivery were taken as 100\%. (d,e) The same methodology as in c was used, except that radiolabelled TZB (15 $\mu \mathrm{g}$ ) injection was followed $24 \mathrm{~h}$ later by intravenous delivery of PBS, HER2-WT (51 $\mu \mathrm{g})$, HER2-Seldeg (51 $\mu \mathrm{g}), \mathrm{MOG}-\mathrm{Seldeg}(31 \mu \mathrm{g})$ or Abdeg (MST-HN; $60 \mu \mathrm{g})$, each at fourfold molar excess. Error bars indicate s.d. and statistically significant differences are indicated for MOG-WT versus MOG-Seldeg (low) (c) HER2-Seldeg versus MOG-Seldeg (d) and HER2-Seldeg versus Abdeg (e) by * $(P<0.05$, two-way analysis of variance with Tukey's multiple comparisons; $n=6$ mice per group). Data shown are representative of two independent experiments. 
the ECD of mouse $\mathrm{MOG}^{23}$ linked to the same engineered $\mathrm{Fc}$ fragment, using a GGGGS linker. Analogous ('wild type') expression constructs were also made to express antigen-Fc fusions without the MST-HN mutations. Sequences of the expression constructs are available upon request.

Protein expression and purification. Recombinant proteins were expressed in HEK-293F (Life Technologies) cells following transient transfection with the Gibco Expi293 expression system kit (Life Technologies). The MST-HN mutations reduce binding of the Fc region to protein G-Sepharose and Seldegs were therefore purified using an anion exchange column (SOURCE-15Q, GE Healthcare) at $\mathrm{pH} 8.0$ and a linear salt gradient $(0-0.5 \mathrm{M} \mathrm{NaCl})$. HER2-WT and MOG-WT were purified using protein G-Sepharose (GE Healthcare). 8-18C5 was expressed in recombinant form and purified using protein G-Sepharose $\mathrm{e}^{23}$, and clinical grade TZB (Herceptin; Roche) was obtained from the UT Southwestern Medical Center Pharmacy. Recombinant Abdeg (MST-HN, hen egg lysozyme-specific) was purified from culture supernatants using lysozyme-Sepharose ${ }^{1}$. All recombinant proteins were purified using size-exclusion chromatography (GE Healthcare) in PBS (Lonza) before use in experiments.

Analyses of Seldeg stability. For serum stability assays, endogenous IgGs were depleted from human male AB plasma (Sigma) by passage through protein G-Sepharose (GE Healthcare). Seldegs were incubated in serum at a concentration of $100 \mathrm{nM}$ at $37^{\circ} \mathrm{C}$ for 3 or 5 days. Following incubation, Seldegs were immunoprecipitated using agarose beads coupled to goat anti-human Fc-specific antibody (Sigma). Immunoprecipitated Seldegs were run on $12 \%$ SDS-polyacrylamide gels, transferred to polyvinylidene difluoride membranes (Millipore) and membranes incubated with horseradish peroxidase-conjugated goat anti-human Fc-specific $(\mathrm{H}+\mathrm{L})$ antibody (Jackson ImmunoResearch). Bound secondary conjugate was detected using Westernsure substrate, followed by scanning with a C-DiGit blot scanner (LI-COR).

Seldegs were also incubated in PBS (Lonza) at $4{ }^{\circ} \mathrm{C}\left(30\right.$ days) or $37^{\circ} \mathrm{C}$ for 5 days, followed by analyses using a Superdex 200 5/150 GL column (GE Healthcare), $12 \%$ SDS-polyacrylamide gel electrophoresis and surface plasmon resonance (BIAcore).

Surface plasmon resonance analyses. Surface plasmon resonance experiments were carried out using a BIAcore T200 (GE Healthcare). To determine the equilibrium binding affinities for the interactions of Seldeg/WT proteins with antibodies (8-18C5 or TZB), 8-18C5 or TZB were injected over immobilized Seldeg/ WT proteins (coupled at $\sim 350-2,000$ relative units on flow cells of CM5 sensor chips) at a flow rate of $10 \mu \mathrm{min}^{-1}$ in PBS (Lonza; $\mathrm{pH} 7.4$ ) plus $0.01 \% \mathrm{v} / \mathrm{v}$ Tween-20. Flow cells were regenerated at the end of each run using $0.15 \mathrm{M} \mathrm{NaCl}$ and $0.1 \mathrm{M}$ glycine $(\mathrm{pH} 2.3)$. The equilibrium dissociation constants $\left(K_{\mathrm{D}} \mathrm{S}\right)$ for the interactions of Seldegs/WT with TZB or 8-18C5 were determined using a 1:1 interaction model and custom-written software ${ }^{24}$. Binding of Seldeg/WT to recombinant human or mouse FcRn in PBS (pH 6.0 or 7.4 ) plus $0.01 \% \mathrm{v} / \mathrm{v}$ Tween-20 was analysed by injecting $100 \mathrm{nM}$ Seldeg/WT over immobilized FcRn (coupled at $\sim 600$ relative units on a CM5 sensor chip) at a flow rate of $10 \mu \mathrm{min}^{-1}$ (ref. 19). The flow cells were regenerated using $0.15 \mathrm{M} \mathrm{NaCl}$ and $0.1 \mathrm{M}$ sodium bicarbonate $(\mathrm{pH} 8.5)$.

Antibody labelling. 8-18C5, TZB, Seldeg, corresponding WT fusion proteins and MST-HN Abdeg were radiolabelled with ${ }^{125} \mathrm{I}$ using Iodogen (Perkin Elmer or MP Biomedicals $)^{25}$. 8-18C5 and TZB were fluorescently labelled with Alexa 647 Fluor (ThermoFisher Scientific) with antibody:dye ratios of 1.6 and 3, respectively. Seldeg/WT fusion proteins were fluorescently labelled with Alexa 555 Fluor (ThermoFisher Scientific) dye at a protein:dye ratio of 2.7 (MOG-WT), 2.5 (MOG-Seldeg), 1.5 (HER2-WT) and 1.2 (HER2-Seldeg) using the manufacturer's protocol.

Fluorescence microscopy and recycling assay. HMEC- 1 cells were transiently co-transfected using Nucleofector technology (Lonza) with human FcRn-GFP $\left(\mathrm{FcRn}\right.$ tagged at the $\mathrm{C}$ terminus with GFP) and human $\beta_{2}$ microglobulin $^{26}$. FcRnGFP containing, a mutated version of human FcRn with similar binding properties as mouse $\mathrm{FcRn}^{19}$, was used throughout these studies.

For microscopy studies, FcRn-GFP-transfected cells were plated in IgGdepleted phenol red-free HAMS F-12K medium on micro-coverglasses (Electron Microscopy Sciences). Eighteen hours post transfection, cells were pulsed with labelled antibodies at a $1: 4$ molar ratio of $8-18 \mathrm{C} 5$ or TZB $\left(15 \mu \mathrm{g} \mathrm{ml}^{-1}\right)$ to MOG- or HER2-Seldeg/WT (31 and $51 \mu \mathrm{g} \mathrm{ml}^{-1}$ for MOG- and HER2-Seldeg/WT, respectively) for $30 \mathrm{~min}$. This pulse was followed by two washes with ice-cold PBS and fixation with $1.7 \%(\mathrm{w} / \mathrm{v})$ paraformaldehyde (Electron Microscopy Sciences) with $0.025 \%(\mathrm{v} / \mathrm{v})$ glutaraldehyde (Sigma) on ice for $10 \mathrm{~min}$. Alternatively, following the $30 \mathrm{~min}$ pulse, cells were washed with PBS and chased in medium for $60 \mathrm{~min}$ before further washes and fixation. To label lysosomes, transfected cells were pre-pulsed with Alexa 555-labelled dextran (ThermoFisher Scientific; $500 \mu \mathrm{g} \mathrm{ml}^{-1}$ ) for $2 \mathrm{~h}$, followed by pulsing with labelled antibodies (as above) for $30 \mathrm{~min}$ and a chase period of $8 \mathrm{~h}$. All pulse-chase experiments were carried out in phenol red-free HAMS F-12K medium $(\mathrm{pH} 7.4)$ at $37^{\circ} \mathrm{C}$ in a $5 \% \mathrm{CO}_{2}$ incubator.
Fixed cells were imaged with a Zeiss Axiovert 200M inverted microscope, and acquired data were analysed using in-house written software (MIATool ${ }^{26}$ ). For recycling assays, transfected HMEC-1 cells were plated in 24-well plates at a density of 50,000 cells per well. Eighteen hours post transfection, cells were pulsed with medium containing labelled antibodies as for the microscopy experiments. At the end of the chase period, cells were washed with ice-cold PBS, followed by trypsinization (PBS and trypsin were maintained at $\mathrm{pH}$ 6.0). Trypsinized cells were collected, fixed on ice for $5 \mathrm{~min}$ with $1.7 \%$ paraformaldehyde (w/v) and analysed using a LSRFortessa flow cytometer (BD Biosciences). Flow cytometry data were analysed using Flowjo (FlowJo).

Studies in mice. All pharmacokinetic experiments were carried out in 8-10-weekold male or female C57BL/6 mice that transgenically express human Fc $\gamma \mathrm{Rs}^{16}$ Animals were housed in a pathogen-free facility at Texas A\&M University and all animal experiments were approved by the Texas A\&M Institutional Animal Care and Use Committee. Mice were injected intravenously with $15 \mu \mathrm{g}$ radiolabelled antibody (8-18C5 or TZB) in $200 \mu \mathrm{l} 0.1 \%$ BSA in PBS (Lonza). Twenty-four hours later, Seldegs and controls ( $n=6$ mice per group) were intravenously delivered in $200 \mu \mathrm{l}$ PBS at a 4 -fold or 16-fold molar excess as indicated in the figure legends. Whole-body radioactive counts were obtained using an Atom Lab 100 dose calibrator (Biodex). Mice were retroorbitally bled using $10 \mu \mathrm{l}$ capillary tubes (Drummond) and radioactive counts (c.p.m.) obtained by gamma counting (Perkin Elmer). To determine total serum IgG levels, mice were retroorbitally bled with $44.7 \mu \mathrm{l}$ heparinized capillary tubes (VWR International) and IgG concentrations in 1:25,000 dilutions of serum in PBS quantified by enzyme-linked immunosorbent assay ${ }^{1}$. All radioactive counts were expressed as the percentage of the levels obtained from serum samples and whole-body counting immediately before Seldeg/control delivery.

To determine the $\beta$-phase half-lives of MOG-WT or -Seldeg, HER2-WT or -Seldeg, and Abdeg (MST-HN), radiolabelled WT/Seldeg or Abdeg were injected ( $n=5$ mice per group) at a dose per mouse of $20 \mu \mathrm{g}$ radiolabelled protein and 10 31 and $40 \mu \mathrm{g}$ of unlabelled proteins for MOG-WT or

-Seldeg, HER2-WT or -Seldeg, and Abdeg, respectively. The $\beta$-phase half-lives for the proteins were determined by fitting the data to a biexponential model ${ }^{25}$.

Statistical analyses. Statistical analyses was carried in GraphPad Prism (GraphPad Software, Inc.) by two-way analysis of variance with Tukey's multiple comparison test.

Data availability. All relevant data and sequences of constructs used in this study are available on request.

\section{References}

1. Vaccaro, C., Zhou, J., Ober, R. J. \& Ward, E. S. Engineering the Fc region of immunoglobulin $\mathrm{G}$ to modulate in vivo antibody levels. Nat. Biotechnol. 23, 1283-1288 (2005).

2. Getman, K. E. \& Balthasar, J. P. Pharmacokinetic effects of 4 C9, an anti-FcRn antibody, in rats: implications for the use of $\mathrm{FcRn}$ inhibitors for the treatment of humoral autoimmune and alloimmune conditions. J. Pharm. Sci. 94, 718-729 (2005).

3. Mezo, A. R. et al. Reduction of IgG in nonhuman primates by a peptide antagonist of the neonatal Fc receptor FcRn. Proc. Natl Acad. Sci. USA 105, 2337-2342 (2008).

4. Liu, L. et al. Amelioration of experimental autoimmune myasthenia gravis in rats by neonatal FcR blockade. J. Immunol. 178, 5390-5398 (2007).

5. Patel, D. A. et al. Neonatal Fc receptor blockade by Fc engineering ameliorates arthritis in a murine model. J. Immunol. 187, 1015-1022 (2011).

6. Bitonti, A. J. et al. Pulmonary delivery of an erythropoietin Fc fusion protein in non-human primates through an immunoglobulin transport pathway. Proc Natl Acad. Sci. USA 101, 9763-9768 (2004).

7. Chu, S. Y. et al. Reduction of total IgE by targeted coengagement of IgE B-cell receptor and FcgammaRIIb with Fc-engineered antibody. J. Allergy Clin. Immunol. 129, 1102-1115 (2012).

8. Ward, E. S. \& Ober, R. J. Chapter 4: multitasking by exploitation of intracellular transport functions the many faces of FcRn. Adv. Immunol. 103, 77-115 (2009).

9. Gan, Z., Ram, S., Vaccaro, C., Ober, R. J. \& Ward, E. S. Analyses of the recycling receptor, $\mathrm{FcRn}$, in live cells reveal novel pathways for lysosomal delivery. Traffic 10, 600-614 (2009).

10. Igawa, T., Haraya, K. \& Hattori, K. Sweeping antibody as a novel therapeutic antibody modality capable of eliminating soluble antigens from circulation. Immunol. Rev. 270, 132-151 (2016).

11. Breithaupt, C. et al. Demyelinating myelin oligodendrocyte glycoproteinspecific autoantibody response is focused on one dominant conformational epitope region in rodents. J. Immunol. 181, 1255-1263 (2008).

12. Lalive, P. H. et al. Antibodies to native myelin oligodendrocyte glycoprotein are serologic markers of early inflammation in multiple sclerosis. Proc. Natl Acad. Sci. USA 103, 2280-2285 (2006). 
13. Zhou, D. et al. Identification of a pathogenic antibody response to native myelin oligodendrocyte glycoprotein in multiple sclerosis. Proc. Natl Acad. Sci. USA 103, 19057-19062 (2006).

14. Carter, P. J. Potent antibody therapeutics by design. Nat. Rev. Immunol. 6, 343-357 (2006)

15. Schluesener, H. J., Sobel, R. A., Linington, C. \& Weiner, H. L. A monoclonal antibody against a myelin oligodendrocyte glycoprotein induces relapses and demyelination in central nervous system autoimmune disease. J. Immunol. 139, 4016-4021 (1987).

16. Smith, P., DiLillo, D. J., Bournazos, S., Li, F. \& Ravetch, J. V. Mouse model recapitulating human $\mathrm{Fc} \gamma$ receptor structural and functional diversity. Proc. Natl Acad. Sci. USA 109, 6181-6186 (2012).

17. Vaccaro, C., Bawdon, R., Wanjie, S., Ober, R. J. \& Ward, E. S. Divergent activities of an engineered antibody in murine and human systems have implications for therapeutic antibodies. Proc. Natl Acad. Sci. USA 103, 18709-18714 (2006).

18. Dall'Acqua, W. F. et al. Increasing the affinity of a human IgG1 for the neonatal Fc receptor: biological consequences. J. Immunol. 169, 5171-5180 (2002).

19. Zhou, J., Mateos, F., Ober, R. J. \& Ward, E. S. Conferring the binding properties of the mouse MHC class I-related receptor, FcRn, onto the human ortholog by sequential rounds of site-directed mutagenesis. J. Mol. Biol. 345, 1071-1081 (2005).

20. Horton, R. M., Hunt, H. D., Ho, S. N., Pullen, J. K. \& Pease, L. R. Engineering hybrid genes without the use of restriction enzymes: gene splicing by overlap extension. Gene 77, 61-68 (1989).

21. Moore, G. L. et al. A novel bispecific antibody format enables simultaneous bivalent and monovalent co-engagement of distinct target antigens. MAbs 3, 546-557 (2011).

22. Merchant, A. M. et al. An efficient route to human bispecific IgG. Nat. Biotechnol. 16, 677-681 (1998).

23. Bansal, P. et al. The encephalitogenic, human myelin oligodendrocyte glycoprotein-induced antibody repertoire is directed toward multiple epitopes in C57BL/6-immunized mice. J. Immunol. 191, 1091-1101 (2013).

24. Ober, R. J. \& Ward, E. S. Compensation for loss of ligand activity in surface plasmon resonance experiments. Anal. Biochem. 306, 228-236 (2002).

25. Montoyo, H. P. et al. Conditional deletion of the MHC class I-related receptor FcRn reveals the sites of IgG homeostasis in mice. Proc. Natl Acad. Sci. USA 106, 2788-2793 (2009).

26. Ober, R. J., Martinez, C., Vaccaro, C., Zhou, J. \& Ward, E. S. Visualizing the site and dynamics of IgG salvage by the MHC class I-related receptor, FcRn. J. Immunol. 172, 2021-2029 (2004).

\section{Acknowledgements}

We are indebted to Drs Jeffrey Ravetch and Patrick Smith for generously providing us with mice transgenic for human Fc $\gamma$ Rs. The HMEC-1 cell line was a generous gift from Francisco Candal (CDC). We thank Joseph J. Heimann and Keerthivasan Ambigapathy for help with plasmid generation and transfections and Kyle Current for maintaining the mouse colony. This work was supported in part by the Cancer Prevention and Research Institute of Texas (RP160051, awarded to E.S. Ward).

\section{Author contributions}

S.C.D., R.J.O. and E.S.W. conceived this study and designed the experiments. S.C.D., P.K. and D.K.C. performed the experiments. S.C.D., R.J.O. and E.S.W. wrote the manuscript.

\section{Additional information}

Supplementary Information accompanies this paper at http://www.nature.com/ naturecommunications

Competing interests: The authors declare no competing financial interests.

Reprints and permission information is available online at http://npg.nature.com/ reprintsandpermissions/

How to cite this article: Devanaboyina, S. C. et al. Engineered clearing agents for the selective depletion of antigen-specific antibodies. Nat. Commun. 8, 15314 doi: $10.1038 /$ ncomms15314 (2017).

Publisher's note: Springer Nature remains neutral with regard to jurisdictional claims in published maps and institutional affiliations.

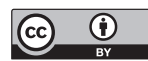

This work is licensed under a Creative Commons Attribution 4.0 International License. The images or other third party material in this article are included in the article's Creative Commons license, unless indicated otherwise in the credit line; if the material is not included under the Creative Commons license, users will need to obtain permission from the license holder to reproduce the material. To view a copy of this license, visit http://creativecommons.org/licenses/by/4.0/

(C) The Author(s) 2017 